International Journal of Engineering \& Technology, $7(3.31)(2018) 98-101$
International Journal of Engineering \& Technology
Website $:$ www.sciencepubco.com/index.php/IJET
Research paper

\title{
Tripled Fixed Point Theorems of Caristi type Contraction in Partially Ordered Metric Space
}

\author{
G.Adilakshmi $^{1}$, G.N.V.Kishore ${ }^{1,2, *}$, N. Veerraju ${ }^{3}$ \\ ${ }^{1}$ Department of Mathematics, K L E F, Vaddeswaram, Guntur, Andhra Pradesh, India - 522502 \\ ${ }^{2,3}$ Department of Engineering Mathematics, S. R. K. R. Engineering College, Bhimavaram, Andhra Pradesh, India-534 204 \\ * Corresponding Author Email: kishore.apr2@gmail.com
}

\begin{abstract}
The main aim of this paper is to obtain a unique common tripled fixed point theorem in partial ordered metric space using Caristi type contraction.
\end{abstract}

Keywords: Partially ordered Metric space, compatible maps, tripled fixed point, Caristi type contraction, lower semi continuous function.

\section{Introduction}

The notion of coupled fixed point is introduced by Bhaskar and Lakshmikantham [1] and studied some fixed point theorems in partially ordered metric spaces. With the inspiration of coupled fixed point theorem concept, In 2011, V.Berinde and M.Borcut [2] introduced the concept of triple fixed point and obtained some fixed point theorems for contraction type maps in partially ordered metric spaces. In 1976, Caristi proved the following famous fixed point theorem.

Theorem 1.1 [6] Let $(\mathrm{X}, \mathrm{d})$ be complete metric space and $\mathrm{f}: \mathrm{X} \rightarrow \mathrm{R}$ be lower semi continuous and bounded below function. A mapping $\mathrm{T}: \mathrm{X} \rightarrow \mathrm{X}$ is said to be Caristi type map on $\mathrm{X}$ dominated by $f$ if $T$ satisfies $d(x, T x) \leq f(x)-f(T x)$ for each $x \in X$. Then $T$ has a fixed point.

It is well-known that the Caristi's fixed point theorem is one of the most valuable generalization of the Banach contraction principle.

The aim of this paper is to study unique common triple fixed point theorem for four maps by using Caristi type contraction for compatible maps over partially ordered Metric Space.

Definition 1.2 (See [1]) Let $(X, \leq)$ be a partially ordered set and $\mathrm{F}: \mathrm{X} \times \mathrm{X} \rightarrow \mathrm{X}$. Then the mapping $\mathrm{F}$ is said to have mixed monotone property if $\mathrm{F}(\mathrm{x}, \mathrm{y})$ is monotone non decreasing in $\mathrm{x}$ and is monotone non increasing in $\mathrm{y}$; that is for any $\mathrm{x}, \mathrm{y} \in \mathrm{X}, \mathrm{x}_{1} \leq \mathrm{x}_{2} \Rightarrow$ $\mathrm{F}\left(\mathrm{x}_{1}, \mathrm{y}\right) \leq \mathrm{F}\left(\mathrm{x}_{2}, \mathrm{y}\right)$ for all $\mathrm{y} \in \mathrm{X}$ and $\mathrm{y}_{1} \leq \mathrm{y}_{2} \Rightarrow \mathrm{F}\left(\mathrm{x}, \mathrm{y}_{2}\right) \leq$ $\mathrm{F}\left(\mathrm{x}, \mathrm{y}_{1}\right)$ for all $\mathrm{x} \in \mathrm{X}$.

Inspired by Definition 1.2, Lakshmikantham and Ciric in [17] introduced the concept of $\mathrm{g}-$ mixed monotone mapping.

Definition 1.3 (See [4]) Let $(X, \leq)$ be a partially ordered set and $\mathrm{F}: \mathrm{X} \times \mathrm{X} \rightarrow \mathrm{X}$. Then the mapping $\mathrm{F}$ is said to have mixed $\mathrm{g}-$ monotone property if $F(x, y)$ is monotone $g$-non decreasing in $X$ and is monotone $\mathrm{g}-$ non increasing in $\mathrm{Y}$; that is for any $\mathrm{x}, \mathrm{y} \in \mathrm{X}$, $\mathrm{gx}_{1} \leq \mathrm{gx}_{2} \Rightarrow \mathrm{F}\left(\mathrm{x}_{1}, \mathrm{y}\right) \leq \mathrm{F}\left(\mathrm{x}_{2}, \mathrm{y}\right)$ for all $\mathrm{y} \in \mathrm{X}$ and $\mathrm{gy}_{1} \leq \mathrm{gy}_{2} \Rightarrow$ $\mathrm{F}\left(\mathrm{x}, \mathrm{y}_{2}\right) \leq \mathrm{F}\left(\mathrm{x}, \mathrm{y}_{1}\right)$ for all $\mathrm{x} \in \mathrm{X}$.
Definition 1.4 (See [3]) Let $F: X \times X \times X \rightarrow X$ and $f: X \rightarrow X$. An element $(\mathrm{x}, \mathrm{y}, \mathrm{z}) \in \mathrm{X} \times \mathrm{X} \times \mathrm{X}$ is called

(i) a coincidence point of mappings $F, f$ if $f x=F(x, y, z), f y=$ $\mathrm{F}(\mathrm{y}, \mathrm{x}, \mathrm{y}), \mathrm{fz}=\mathrm{F}(\mathrm{z}, \mathrm{y}, \mathrm{x})$.

(ii) a common tripled fixed point of the mappings $F, f$ if $x=f x=$ $\mathrm{F}(\mathrm{x}, \mathrm{y}, \mathrm{z}), \mathrm{y}=\mathrm{fy}=\mathrm{F}(\mathrm{y}, \mathrm{x}, \mathrm{y}), \mathrm{z}=\mathrm{fz}=\mathrm{F}(\mathrm{z}, \mathrm{y}, \mathrm{x})$.

Definition 1.5 (See [3]) The mappings $F: X \times X \times X \rightarrow X$ and $\mathrm{f}: \mathrm{X} \rightarrow \mathrm{X}$ are said to be commutative if $\mathrm{f}(\mathrm{F}(\mathrm{x}, \mathrm{y}, \mathrm{z}))=$ $F(f x, f y, f z), f(F(y, x, y))=F(f y, f x, f y) \quad$ and $\quad f(F(z, y, x))=$ $F(f z, f y, f x)$.

Lemma 1.6 (See [5]) Let $\triangleleft$ be a reflexive relation on a nonempty set $\mathrm{M}$ and $\phi: \mathrm{M} \rightarrow \mathrm{R}$ a function bounded from below, then $\chi \triangleleft \gamma$ and $\chi \neq \gamma$; then $\phi(\chi)>\phi(\gamma)$.

\section{Main Results}

Theorem 2.1: Let $(X, \leq, d)$ be a partially ordered metric space and let $\mathrm{S}, \mathrm{T}: \mathrm{X} \times \mathrm{X} \times \mathrm{X} \rightarrow \mathrm{X}$ and $\mathrm{f}, \mathrm{g}: \mathrm{X} \rightarrow \mathrm{X}$ be two mappings satisfies

$$
\begin{aligned}
& \text { (2.1.1) } d(S(x, y, z), T(u, v, w)) \\
& \left.\leq \max \left\{\begin{array}{l}
\zeta(\max (\eta(f x, g u), \eta(f y, g v), \eta(f z, g w))), \\
\max \left(\begin{array}{c}
\eta(S(x, y, z), T(u, v, w)), \\
\eta(S(y, x, y), T(v, u, v)), \\
\eta(S(z, y, x), T(w, v, u))
\end{array}\right)
\end{array}\right)\right\} \\
& \max (\eta(f x, g u), \eta(f y, g v), \eta(f z, g w)) \\
& -\max \left(\begin{array}{l}
\eta(S(x, y, z), T(u, v, w)), \\
\eta(S(y, x, y), T(v, u, v)), \\
\eta(S(z, y, x), T(w, v, u))
\end{array}\right)
\end{aligned}
$$


where $\eta: X \times X \rightarrow[0, \infty)$ is lower semi continuous function and $\zeta:[0, \infty) \rightarrow[0, \infty)$ be an upper semi continuous function.

(2.1.2) $\mathrm{S}(\mathrm{X} \times \mathrm{X} \times \mathrm{X}) \subseteq \mathrm{g}(\mathrm{X})$ and $\mathrm{T}(\mathrm{X} \times \mathrm{X} \times \mathrm{X}) \subseteq \mathrm{f}(\mathrm{X})$,

(2.1.3) $\mathrm{S}$ has the mixed $\mathrm{g}-$ monotone property and $\mathrm{T}$ has the mixed $\mathrm{f}-$ monotone property.

(2.1.4) f, g are continuous and f commutes with $\mathrm{S}$, g commutes with $\mathrm{T}$,

(2.1.5) either $f(X)$ or $g(X)$ is complete.

(2.1.6) (a) if a non decreasing sequence $\left\{x_{n}\right\} \rightarrow x$, then $x_{n} \leq x$ for all $n$,

(b) if a non increasing sequence $\left\{x_{n}\right\} \rightarrow x$, then $x \leq x_{n}$ for all $n$, for $\mathrm{x}_{0}, \mathrm{y}_{0}, \mathrm{z}_{0} \in \mathrm{X}$ there exists $\mathrm{x}_{1}, \mathrm{y}_{1}, \mathrm{z}_{1} \in \mathrm{X}$ such that

$\mathrm{gx}_{0} \geq \mathrm{S}\left(\mathrm{x}_{0}, \mathrm{y}_{0}, \mathrm{z}_{0}\right), \mathrm{gy}_{0} \leq \mathrm{S}\left(\mathrm{y}_{0}, \mathrm{x}_{0}, \mathrm{y}_{0}\right), \mathrm{gz}_{0} \geq \mathrm{S}\left(\mathrm{z}_{0}, \mathrm{y}_{0}, \mathrm{x}_{0}\right)$ and

$\mathrm{fx}_{1} \geq \mathrm{T}\left(\mathrm{x}_{1}, \mathrm{y}_{1}, \mathrm{z}_{1}\right), \mathrm{fy}_{1} \leq \mathrm{T}\left(\mathrm{y}_{1}, \mathrm{x}_{1}, \mathrm{y}_{1}\right), \mathrm{fz}_{1} \geq \mathrm{T}\left(\mathrm{z}_{1}, \mathrm{y}_{1}, \mathrm{x}_{1}\right)$ then

$\mathrm{S}, \mathrm{T}, \mathrm{f}, \mathrm{g}$ have unique common tripled fixed point.

Proof: Let $\mathrm{x}_{0}, \mathrm{y}_{0}, \mathrm{z}_{0} \in \mathrm{X}$ be arbitrary points in $\mathrm{X}$. From (2.1.2) and (2.1.6), there exist sequences $\left\{x_{2 n}\right\},\left\{y_{2 n}\right\},\left\{z_{2 n}\right\}$ and $\left\{P_{2 n}\right\}$, $\left\{Q_{2 n}\right\},\left\{R_{2 n}\right\}$ in $X$ such that

$P_{2 n}=g x_{2 n+1}=S\left(x_{2 n}, y_{2 n}, z_{2 n}\right)$,

$\mathrm{Q}_{2 \mathrm{n}}=\mathrm{gy}_{2 \mathrm{n}+1}=\mathrm{S}\left(\mathrm{y}_{2 \mathrm{n}}, \mathrm{x}_{2 \mathrm{n}}, \mathrm{y}_{2 \mathrm{n}}\right)$,

$\mathrm{R}_{2 \mathrm{n}}=\mathrm{gz}_{2 \mathrm{n}+1}=\mathrm{S}\left(\mathrm{z}_{2 \mathrm{n}}, \mathrm{y}_{2 \mathrm{n}}, \mathrm{x}_{2 \mathrm{n}}\right)$,

$\mathrm{P}_{2 n+1}=\mathrm{fx}_{2 \mathrm{n}+2}=\mathrm{T}\left(\mathrm{x}_{2 \mathrm{n}+1}, \mathrm{y}_{2 \mathrm{n}+1}, \mathrm{z}_{2 \mathrm{n}+1}\right)$,

$\mathrm{Q}_{2 \mathrm{n}+1}=\mathrm{fy}_{2 \mathrm{n}+2}=\mathrm{T}\left(\mathrm{y}_{2 \mathrm{n}+1}, \mathrm{x}_{2 \mathrm{n}+1}, \mathrm{y}_{2 \mathrm{n}+1}\right)$,

$\mathrm{R}_{2 \mathrm{n}+1}=\mathrm{fz}_{2 \mathrm{n}+2}=\mathrm{T}\left(\mathrm{z}_{2 \mathrm{n}+1}, \mathrm{y}_{2 \mathrm{n}+1}, \mathrm{x}_{2 \mathrm{n}+1}\right), \quad \mathrm{n}=0,1,2, \cdots$

with

$\mathrm{gx}_{1} \geq \mathrm{gx}_{2} \geq \mathrm{gx}_{3} \geq \mathrm{gx}_{4} \cdots$

$\mathrm{gy}_{1} \leq \mathrm{gy}_{2} \leq \mathrm{gy}_{3} \leq \mathrm{gy}_{4} \cdots$

$\mathrm{gz}_{1} \geq \mathrm{gz}_{2} \geq \mathrm{gz}_{3} \geq \mathrm{gz}_{4} \cdots$

and

$\mathrm{fx}_{2} \geq \mathrm{fx}_{3} \geq \mathrm{fx}_{4} \geq \mathrm{fx}_{5} \cdots$

$\mathrm{fy}_{2} \geq \mathrm{fy}_{3} \geq \mathrm{fy}_{4} \geq \mathrm{fy}_{5} \cdots$

$\mathrm{fz}_{2} \geq \mathrm{fz}_{3} \geq \mathrm{fz}_{4} \geq \mathrm{fz}_{5} \cdots$

Define a relation $\triangleleft$ on $\mathrm{X}$ as follows:

$\mathrm{S}(\mathrm{x}, \mathrm{y}, \mathrm{z}) \triangleleft \mathrm{T}(\mathrm{u}, \mathrm{v}, \mathrm{w}) \Leftrightarrow \mathrm{d}(\mathrm{S}(\mathrm{x}, \mathrm{y}, \mathrm{z}), \mathrm{T}(\mathrm{u}, \mathrm{v}, \mathrm{w}))$

$\leq \max \left\{\begin{array}{l}\zeta(\max (\eta(f x, g u), \eta(f y, g v), \eta(f z, g w))), \\ \zeta\left(\max \left(\begin{array}{c}\eta(S(x, y, z), T(u, v, w)), \\ \eta(S(y, x, y), T(v, u, v)), \\ \eta(S(z, y, x), T(w, v, u))\end{array}\right)\right)\end{array}\right\}$

$\max (\eta(f x, g u), \eta(f y, g v), \eta(f z, g w))$

$$
\left.-\max \left(\begin{array}{c}
\eta(S(x, y, z), T(u, v, w)) \\
\eta(S(y, x, y), T(v, u, v)) \\
\eta(S(z, y, x), T(w, v, u))
\end{array}\right)\right]
$$

Then clearly $\triangleleft$ is a reflexive relation on $\mathrm{X}$.

$$
\begin{aligned}
& \text { Now for } P_{2 n} \neq P_{2 n+1} \\
& d\left(P_{2 n}, P_{2 n+1}\right) \\
& =d\left(S\left(x_{2 n}, y_{2 n}, z_{2 n}\right), T\left(x_{2 n+1}, y_{2 n+1}, z_{2 n+1}\right)\right) \\
& \leq \max \left\{\begin{array}{c}
\zeta\left(\max \left(\eta\left(P_{2 n-1}, P_{2 n}\right), \eta\left(Q_{2 n-1}, Q_{2 n}\right), \eta\left(R_{2 n-1}, R_{2 n}\right)\right)\right), \\
\zeta\left(\max \left(\eta\left(P_{2 n}, P_{2 n+1}\right), \eta\left(Q_{2 n}, Q_{2 n+1}\right), \eta\left(R_{2 n}, R_{2 n+1}\right)\right)\right)
\end{array}\right\}
\end{aligned}
$$$$
\left[\begin{array}{c}
\max \left(\eta\left(P_{2 n-1}, P_{2 n}\right), \eta\left(Q_{2 n-1}, Q_{2 n}\right), \eta\left(R_{2 n-1}, R_{2 n}\right)\right) \\
-\max \left(\eta\left(P_{2 n}, P_{2 n+1}\right), \eta\left(Q_{2 n}, Q_{2 n+1}\right), \eta\left(R_{2 n}, R_{2 n+1}\right)\right)
\end{array}\right]
$$

In this way for $Q_{2 n} \neq Q_{2 n+1}$
$d\left(Q_{2 n}, Q_{2 n+1}\right)$

$\leq \max \left\{\begin{array}{l}\zeta\left(\max \left(\eta\left(P_{2 n-1}, P_{2 n}\right), \eta\left(Q_{2 n-1}, Q_{2 n}\right), \eta\left(R_{2 n-1}, R_{2 n}\right)\right)\right), \\ \zeta\left(\max \left(\eta\left(P_{2 n}, P_{2 n+1}\right), \eta\left(Q_{2 n}, Q_{2 n+1}\right), \eta\left(R_{2 n}, R_{2 n+1}\right)\right)\right)\end{array}\right\}$

$$
\left[\begin{array}{c}
\max \left(\eta\left(P_{2 n-1}, P_{2 n}\right), \eta\left(Q_{2 n-1}, Q_{2 n}\right), \eta\left(R_{2 n-1}, R_{2 n}\right)\right) \\
-\max \left(\eta\left(P_{2 n}, P_{2 n+1}\right), \eta\left(Q_{2 n}, Q_{2 n+1}\right), \eta\left(R_{2 n}, R_{2 n+1}\right)\right)
\end{array}\right]
$$

and $\mathrm{R}_{2 \mathrm{n}} \neq \mathrm{QR}_{2 \mathrm{n}+1}$

$d\left(R_{2 n}, R_{2 n+1}\right)$

$\leq \max \left\{\begin{array}{l}\zeta\left(\max \left(\eta\left(P_{2 n-1}, P_{2 n}\right), \eta\left(Q_{2 n-1}, Q_{2 n}\right), \eta\left(R_{2 n-1}, R_{2 n}\right)\right)\right), \\ \zeta\left(\max \left(\eta\left(P_{2 n}, P_{2 n+1}\right), \eta\left(Q_{2 n}, Q_{2 n+1}\right), \eta\left(R_{2 n}, R_{2 n+1}\right)\right)\right)\end{array}\right\}$

$$
\left[\begin{array}{c}
\max \left(\eta\left(P_{2 n-1}, P_{2 n}\right), \eta\left(Q_{2 n-1}, Q_{2 n}\right), \eta\left(R_{2 n-1}, R_{2 n}\right)\right) \\
-\max \left(\eta\left(P_{2 n}, P_{2 n+1}\right), \eta\left(Q_{2 n}, Q_{2 n+1}\right), \eta\left(R_{2 n}, R_{2 n+1}\right)\right)
\end{array}\right]
$$

Therefore

$$
\begin{aligned}
\max \left\{d\left(P_{2 n}, P_{2 n+1}\right), d\left(Q_{2 n}, Q_{2 n+1}\right), d\left(R_{2 n}, R_{2 n+1}\right)\right\} \\
\leq \max \left\{\begin{array}{l}
\zeta\left(\max \left(\eta\left(P_{2 n-1}, P_{2 n}\right), \eta\left(Q_{2 n-1}, Q_{2 n}\right), \eta\left(R_{2 n-1}, R_{2 n}\right)\right)\right), \\
\zeta\left(\max \left(\eta\left(P_{2 n}, P_{2 n+1}\right), \eta\left(Q_{2 n}, Q_{2 n+1}\right), \eta\left(R_{2 n}, R_{2 n+1}\right)\right)\right)
\end{array}\right\} \\
{\left[\begin{array}{c}
\max \left(\eta\left(P_{2 n-1}, P_{2 n}\right), \eta\left(Q_{2 n-1}, Q_{2 n}\right), \eta\left(R_{2 n-1}, R_{2 n}\right)\right) \\
-\max \left(\eta\left(P_{2 n}, P_{2 n+1}\right), \eta\left(Q_{2 n}, Q_{2 n+1}\right), \eta\left(R_{2 n}, R_{2 n+1}\right)\right)
\end{array}\right] }
\end{aligned}
$$

Since for $P_{2 n} \neq P_{2 n+1}$ we have $P_{2 n} \triangleleft P_{2 n+1}$, for $Q_{2 n} \neq Q_{2 n+1}$ we have $Q_{2 n} \triangleleft Q_{2 n+1}$ and for $R_{2 n} \neq R_{2 n+1}$ we have $R_{2 n} \triangleleft R_{2 n+1}$. Therefore from Lemma 1.6 we have $\left\{\eta\left(P_{2 n}\right), \eta\left(P_{2 n+1}\right)\right\}$ and $\left\{\eta\left(Q_{2 n}\right), \eta\left(Q_{2 n+1}\right)\right\},\left\{\eta\left(R_{2 n}\right), \eta\left(R_{2 n+1}\right)\right\}$, are non increasing. Let $\lim _{\mathrm{n} \rightarrow \infty}\left\{\eta\left(\mathrm{P}_{2 \mathrm{n}}\right), \eta\left(\mathrm{P}_{2 \mathrm{n}+1}\right)\right\}=\lambda_{1}, \lim _{\mathrm{n} \rightarrow \infty}\left\{\eta\left(\mathrm{Q}_{2 \mathrm{n}}\right), \eta\left(\mathrm{Q}_{2 \mathrm{n}+1}\right)\right\}=$ $\lambda_{2}$,

$\lim _{n \rightarrow \infty}\left\{\eta\left(R_{2 n}\right), \eta\left(R_{2 n+1}\right)\right\}=\lambda_{3}$. for some $\lambda_{1}, \lambda_{2}, \lambda_{3} \geq 0$.

If $\lambda_{1}, \lambda_{2}, \lambda_{3}=0$ then we get a contradiction .

So $\lambda_{1}, \lambda_{2}, \lambda_{3}>0$.

Since $\zeta$ is upper semi continuous function so we have

$\lim _{n \rightarrow \infty} \sup \zeta\left(\left\{\eta\left(P_{2 n}\right), \eta\left(P_{2 n+1}\right)\right\}\right)=\zeta\left(\lambda_{1}\right)$,

$\lim _{n \rightarrow \infty} \sup \zeta\left(\left\{\eta\left(Q_{2 n}\right), \eta\left(Q_{2 n+1}\right)\right\}\right)=\zeta\left(\lambda_{2}\right)$,

$\lim _{n \rightarrow \infty} \sup \zeta\left(\left\{\eta\left(R_{2 n}\right), \eta\left(R_{2 n+1}\right)\right\}\right)=\zeta\left(\lambda_{3}\right)$.

So for any $m \in N$ with $n \geq n_{0}$ we have

$\lim _{n \rightarrow \infty} \sup \zeta\left(\left\{\eta\left(P_{2 n}\right), \eta\left(P_{2 n+1}\right)\right\}\right)=\zeta\left(\lambda_{1}\right)+1$,

$\lim _{n \rightarrow \infty} \sup \zeta\left(\left\{\eta\left(Q_{2 n}\right), \eta\left(Q_{2 n+1}\right)\right\}\right)=\zeta\left(\lambda_{2}\right)+1$,

$\lim _{n \rightarrow \infty} \sup \zeta\left(\left\{\eta\left(R_{2 n}\right), \eta\left(R_{2 n+1}\right)\right\}\right)=\zeta\left(\lambda_{3}\right)+1$.

Therefore

$\max \left\{d\left(P_{2 n}, P_{2 n+1}\right), d\left(Q_{2 n}, Q_{2 n+1}\right), d\left(R_{2 n}, R_{2 n+1}\right)\right\}$

$$
\begin{aligned}
& \leq \max \left[\zeta\left(\lambda_{1}\right)+1, \zeta\left(\lambda_{2}\right)+1, \zeta\left(\lambda_{3}\right)+1\right] \\
& \quad\left[\begin{array}{c}
\max \left(\eta\left(P_{2 n-1}, P_{2 n}\right), \eta\left(Q_{2 n-1}, Q_{2 n}\right), \eta\left(R_{2 n-1}, R_{2 n}\right)\right) \\
-\max \left(\eta\left(P_{2 n}, P_{2 n+1}\right), \eta\left(Q_{2 n}, Q_{2 n+1}\right), \eta\left(R_{2 n}, R_{2 n+1}\right)\right)
\end{array}\right]
\end{aligned}
$$

As $\mathrm{n} \rightarrow \infty$,

$\max \left\{d\left(P_{2 n}, P_{2 n+1}\right), d\left(Q_{2 n}, Q_{2 n+1}\right), d\left(R_{2 n}, R_{2 n+1}\right)\right\} \rightarrow 0$.

Now for $m>n$, and as $m, n \rightarrow \infty$

$\max \left\{d\left(P_{2 n}, P_{2 n+1}\right), d\left(Q_{2 n}, Q_{2 n+1}\right), d\left(R_{2 n}, R_{2 n+1}\right)\right\}+$ $\max \left\{d\left(P_{2 n+1}, P_{2 n+2}\right), d\left(Q_{2 n+1}, Q_{2 n+2}\right), d\left(R_{2 n+1}, R_{2 n+2}\right)\right\}+\cdots+$ $\max \left\{\mathrm{d}\left(\mathrm{P}_{2 \mathrm{~m}}, \mathrm{P}_{2 \mathrm{~m}+1}\right), \mathrm{d}\left(\mathrm{Q}_{2 \mathrm{~m}}, \mathrm{Q}_{2 \mathrm{~m}+1}\right), \mathrm{d}\left(\mathrm{R}_{2 \mathrm{~m}}, \mathrm{R}_{2 \mathrm{~m}+1}\right)\right\}=0$.

This shows $\left\{P_{2 n}\right\},\left\{Q_{2 n}\right\},\left\{R_{2 n}\right\}$ are Cauchy sequence in $X$. Since $f(X)$ is a complete subspace of $(X, d)$ so $\left\{P_{2 n}\right\} \subseteq f(X)$, $\left\{\mathrm{Q}_{2 \mathrm{n}}\right\} \subseteq \mathrm{f}(\mathrm{X})$ and $\left\{\mathrm{R}_{2 \mathrm{n}}\right\} \subseteq \mathrm{f}(\mathrm{X})$ are converges in the complete metric space $(\mathrm{f}(\mathrm{X}), \mathrm{d})$.

Therefore $\lim _{n \rightarrow \infty} d\left(P_{2 n}, u\right)=0, \lim _{n \rightarrow \infty} d\left(Q_{2 n}, v\right)=0$ and $\lim _{n \rightarrow \infty} d\left(R_{2 n}, w\right)=0$ where $u, v, w \in f(X)$. 
Since $f: X \rightarrow X$ and $u, v, w \in f(X)$ so there exists $s_{1}, s_{2}, s_{3} \in X$ such that $\mathrm{fs}_{1}=\mathrm{u}, \mathrm{fs}_{2}=\mathrm{v}$ and $\mathrm{fs}_{3}=\mathrm{w}$.

Since as $\mathrm{n} \rightarrow \infty, \mathrm{S}\left(\mathrm{x}_{2 \mathrm{n}}, \mathrm{y}_{2 \mathrm{n}}, \mathrm{z}_{2 \mathrm{n}}\right) \rightarrow \mathrm{u}$ and $\mathrm{f}$ is continuous and $(\mathrm{s}, \mathrm{f})$ are commute so

$\mathrm{f}\left(\mathrm{S}\left(\mathrm{x}_{2 \mathrm{n}}, \mathrm{y}_{2 \mathrm{n}}, \mathrm{z}_{2 \mathrm{n}}\right)\right) \rightarrow \mathrm{fu}$

$\Rightarrow \mathrm{S}\left(\mathrm{fx}_{2 \mathrm{n}}, \mathrm{fy}_{2 \mathrm{n}}, \mathrm{fz}_{2 \mathrm{n}}\right) \rightarrow \mathrm{fu}$

$\Rightarrow \mathrm{S}(\mathrm{u}, \mathrm{v}, \mathrm{w})=\mathrm{fu}$.

In the same way we can prove that,

$\mathrm{S}(\mathrm{v}, \mathrm{u}, \mathrm{v})=\mathrm{fv}, \mathrm{S}(\mathrm{w}, \mathrm{v}, \mathrm{u})=\mathrm{fw}$.

Also, as $\mathrm{n} \rightarrow \infty, \mathrm{T}\left(\mathrm{x}_{2 \mathrm{n}+1}, \mathrm{y}_{2 \mathrm{n}+1}, \mathrm{z}_{2 \mathrm{n}+1}\right) \rightarrow \mathrm{u}$ and $\mathrm{g}$ is continuous and $(\mathrm{T}, \mathrm{g})$ are commute so

$\mathrm{g}\left(\mathrm{T}\left(\mathrm{x}_{2 \mathrm{n}+1}, \mathrm{y}_{2 \mathrm{n}+1}, \mathrm{z}_{2 \mathrm{n}+1}\right)\right) \rightarrow \mathrm{gu}$

$\Rightarrow \mathrm{T}\left(\mathrm{gx}_{2 \mathrm{n}+1}, \mathrm{gy}_{2 \mathrm{n}+1}, \mathrm{gz}_{2 \mathrm{n}+1}\right) \rightarrow \mathrm{gu}$

$\Rightarrow \mathrm{T}(\mathrm{u}, \mathrm{v}, \mathrm{w})=\mathrm{gu}$.

In the same way we can prove that,

$\mathrm{T}(\mathrm{v}, \mathrm{u}, \mathrm{v})=\mathrm{gv}, \mathrm{S}(\mathrm{w}, \mathrm{v}, \mathrm{u})=\mathrm{gw}$.

Now

$\mathrm{d}(\mathrm{fu}, \mathrm{gu})$

$=\mathrm{d}(\mathrm{S}(\mathrm{u}, \mathrm{v}, \mathrm{w}), \mathrm{T}(\mathrm{u}, \mathrm{v}, \mathrm{w}))$

$\leq \zeta(\max (\eta(f u, g u), \eta(f v, g v), \eta(f w, g v))$

$$
\left\{\begin{array}{c}
\max (\eta(f u, g u), \eta(f v, g v), \eta(f w, g w)) \\
-\max (\eta(f u, g u), \eta(f v, g v), \eta(f w, g w))
\end{array}\right\}
$$

$=0$

Therefore $\mathrm{fu}=\mathrm{gu}$.

Similarly we get $\mathrm{fv}=\mathrm{gv}, \mathrm{fw}=\mathrm{gw}$.

Hence

$\mathrm{fu}=\mathrm{gu}=\mathrm{S}(\mathrm{u}, \mathrm{v}, \mathrm{w})=\mathrm{T}(\mathrm{u}, \mathrm{v}, \mathrm{w})$,

$f v=g v=S(v, u, v)=T(v, u, v)$ and

$\mathrm{fw}=\mathrm{gw}=\mathrm{S}(\mathrm{w}, \mathrm{v}, \mathrm{u})=\mathrm{T}(\mathrm{w}, \mathrm{v}, \mathrm{u})$.

This shows that $(u, v, w)$ is tripled coincidence point of $S, T, f$ and g. Now to prove that $(\mathrm{u}, \mathrm{v}, \mathrm{w})$ is a fixed point of $\mathrm{S}, \mathrm{T}, \mathrm{f}$ and $\mathrm{g}$.

Consider

$\mathrm{d}\left(\mathrm{fu}, \mathrm{z}_{2 \mathrm{n}}\right)$

$=\mathrm{d}\left(\mathrm{S}(\mathrm{u}, \mathrm{v}, \mathrm{w}), \mathrm{T}\left(\mathrm{x}_{2 \mathrm{n}}, \mathrm{y}_{2 \mathrm{n}}, \mathrm{z}_{2 \mathrm{n}}\right)\right)$

$\leq \max \left\{\begin{array}{c}\zeta\left(\max \left(\eta\left(f u, g x_{2 n}\right), \eta\left(f v, g_{2 n}\right), \eta\left(f z, g_{2 n}\right)\right)\right), \\ \zeta\left(\max \left(\eta\left(S(u, v, w), P_{2 n-1}\right), \eta\left(S(v, u, v), Q_{2 n-1}\right),\right.\right.\end{array}\right\}$

$\left[\begin{array}{c}\max \left(\eta\left(f u, g x_{2 n}\right), \eta\left(f v, g y_{2 n}\right), \eta\left(f z, g z_{2 n}\right)\right), \\ -\max \left(\eta\left(S(u, v, w), P_{2 n-1}\right), \eta\left(S(v, u, v), Q_{2 n-1}\right),\right. \\ \left.\eta\left(S(w, v, u), R_{2 n-1}\right)\right)\end{array}\right]$

$\leq \max \left\{\begin{array}{c}\zeta\left(\max \left(\eta\left(f u, g x_{2 n}\right), \eta\left(f v, g y_{2 n}\right), \eta\left(f z, g z_{2 n}\right)\right)\right), \\ \zeta\left(\max \left(\eta\left(f u, P_{2 n-1}\right), \eta\left(f v, Q_{2 n-1}\right), \eta\left(f w, R_{2 n-1}\right)\right)\right)\end{array}\right\}$

$\left[\begin{array}{c}\max \left(\eta\left(f u, g x_{2 n}\right), \eta\left(f v, g y_{2 n}\right), \eta\left(f z, g z_{2 n}\right)\right) \\ -\max \left(\eta\left(f u, P_{2 n-1}\right), \eta\left(f v, Q_{2 n-1}\right), \eta\left(f w, R_{2 n-1}\right)\right)\end{array}\right]$

Letting $\mathrm{n} \rightarrow \infty$, we have that

$d(f u, u) \rightarrow 0$. Therefore $f u=u$.

Similarly we can prove $f v=v$ and $f w=w$. Now it remains to prove the uniqueness of $(\mathrm{u}, \mathrm{v}, \mathrm{w})$.

Suppose $\left(\mathrm{u}^{*}, \mathrm{v}^{*}, \mathrm{w}^{*}\right)$ be an another coupled fixed point of $\mathrm{S}, \mathrm{T}, \mathrm{f}$ and $g$.

$$
\begin{gathered}
f u=u=S(u, v, w), u^{*}=S\left(u^{*}, v^{*}, w^{*}\right)=f u^{*}, \\
f v=v=S(v, u, v), v^{*}=S\left(v^{*}, u^{*}, v^{*}\right)=f v^{*}, \\
f w=w=S(w, v, u), w^{*}=S\left(w^{*}, v^{*}, u^{*}\right)=f w^{*}, \\
g u=u=T(u, v, w), u^{*}=T\left(u^{*}, v^{*}, w^{*}\right)=g u^{*}, \\
g v=v=T(v, u, v), v^{*}=T\left(v^{*}, u^{*}, v^{*}\right)=g v^{*}, \\
g w=w=T(w, v, u), w^{*}=T\left(w^{*}, v^{*}, u^{*}\right)=g w^{*} .
\end{gathered}
$$

Now

$d\left(u, u^{*}\right)=d\left(S(u, v, w), T\left(u^{*}, v^{*}, w^{*}\right)\right)$

$$
\begin{aligned}
& \leq \max \left(\zeta\left(\max \left(\eta\left(u, u^{*}\right), \eta\left(v, v^{*}\right), \eta\left(w, w^{*}\right)\right)\right)\right) \\
& {\left[\begin{array}{c}
\max \left(\eta\left(u, u^{*}\right), \eta\left(v, v^{*}\right), \eta\left(w, w^{*}\right)\right) \\
-\max \left(\eta\left(u, u^{*}\right), \eta\left(v, v^{*}\right), \eta\left(w, w^{*}\right)\right)
\end{array}\right]} \\
& =0 .
\end{aligned}
$$

Therefore $\mathrm{u}=\mathrm{u}^{*}$.

Similarly we can prove that $\mathrm{v}=\mathrm{v}^{*}$ and $\mathrm{w}=\mathrm{w}^{*}$.

Hence the results is proved.

Example 2.2 Let $X=[0,1]$ and define $d: X \times X \rightarrow R^{+}$as $\mathrm{d}(\mathrm{x}, \mathrm{y})=\frac{2}{3}(\mathrm{x}-\mathrm{y})^{2}$ and define $\mathrm{S}, \mathrm{T}: \mathrm{X} \times \mathrm{X} \times \mathrm{X} \rightarrow \mathrm{X}$ as $\mathrm{S}(\mathrm{x}, \mathrm{y}, \mathrm{z})=$ $\frac{x y z}{4}$ and $T(x, y, z)=\frac{x^{2} y^{2} z^{2}}{3}$ and define $f, g: X \rightarrow X$ as $g x=x, f x=\frac{x}{2}$. Define a lower semi continuous function $\eta: X \times X \rightarrow[0, \infty)$ as $\eta(s, t)=s+t$, define $\zeta(a)=\frac{a}{2}$ for all $a \in X$.

Then clearly $S, T, f, g$ satisfies the all the conditions of Theorem 2.1 except condition (2.1.1).

Then $\mathrm{S}, \mathrm{T}, \mathrm{f}$ and $\mathrm{g}$ have a unique common coupled fixed point if they satisfies the condition (2.1.1) of Theorem 2.1.

Now consider,

R.H.S

$=\max \left\{\zeta\left(\max \left(\frac{\mathrm{x}}{2}+\mathrm{u}, \frac{\mathrm{y}}{2}+\mathrm{v}, \frac{\mathrm{z}}{2}+\mathrm{w}\right)\right), \zeta\left(\frac{\mathrm{xyz}}{4}+\frac{\mathrm{u}^{2} \mathrm{v}^{2} \mathrm{w}^{2}}{3}\right)\right\}$

$\left[\max \left(\frac{\mathrm{x}}{2}+\mathrm{u}, \frac{\mathrm{y}}{2}+\mathrm{v}, \frac{\mathrm{z}}{2}+\mathrm{w}\right)-\frac{\mathrm{xyz}}{4}-\frac{\mathrm{u}^{2} \mathrm{v}^{2} \mathrm{w}^{2}}{3}\right]$

$=\frac{1}{2}\left(\frac{x y z}{4}+\frac{u^{2} v^{2} w^{2}}{3}\right)\left[\max \left(\frac{x}{2}+u, \frac{y}{2}+v, \frac{z}{2}+w\right)-\frac{x y z}{4}-\frac{u^{2} v^{2} w^{2}}{3}\right]$

$>\frac{2}{3}\left(\frac{\mathrm{xyz}}{4}-\frac{\mathrm{u}^{2} \mathrm{v}^{2} \mathrm{z}^{2}}{3}\right)^{2}$

$=\mathrm{d}\left(\frac{\mathrm{xyz}}{4}, \frac{\mathrm{u}^{2} \mathrm{v}^{2} \mathrm{z}^{2}}{3}\right)$

$=\mathrm{d}(\mathrm{S}(\mathrm{x}, \mathrm{y}, \mathrm{z}), \mathrm{T}(\mathrm{u}, \mathrm{v}, \mathrm{w}))=$ L.H.S, for all $\mathrm{x}, \mathrm{y}, \mathrm{z}, \mathrm{u}, \mathrm{v}, \mathrm{w} \in(0,1]$

Therefore we have L.H.S $<$ R.H.S

Hence by Theorem $2.1 \mathrm{~S}, \mathrm{~T}, \mathrm{f}$, g have a unique common coupled fixed point.

Corollary 1: Let $(X, \leq, d)$ be a partially ordered metric space and let $\mathrm{S}: \mathrm{X} \times \mathrm{X} \times \mathrm{X} \rightarrow \mathrm{X}$ and $\mathrm{f}: \mathrm{X} \rightarrow \mathrm{X}$ be two mappings satisfies (1.1) $d(S(x, y, z), S(u, v, w))$

$$
\leq \max \left\{\begin{array}{l}
\zeta(\max (\eta(f x, f u), \eta(f y, f v), \eta(f z, f w))), \\
\zeta\left(\max \left(\begin{array}{c}
\eta(S(x, y, z), S(u, v, w)), \\
\eta(S(y, x, y), S(v, u, v)), \\
\eta(S(z, y, x), S(w, v, u))
\end{array}\right)\right)
\end{array}\right\}
$$

$\max (\eta(f x, f u), \eta(f y, f v), \eta(f z, f w))$

$$
\left.-\max \left(\begin{array}{c}
\eta(S(x, y, z), S(u, v, w)) \\
\eta(S(y, x, y), S(v, u, v)) \\
\eta(S(z, y, x), S(w, v, u))
\end{array}\right)\right]
$$

where $\eta: X \times X \rightarrow[0, \infty)$ is lower semi continuous function and $\zeta:[0, \infty) \rightarrow[0, \infty)$ be an upper semi continuous function.

(2.1.2) $\mathrm{S}(\mathrm{X} \times \mathrm{X} \times \mathrm{X}) \subseteq \mathrm{f}(\mathrm{X})$,

(2.1.3) $\mathrm{S}$ has the mixed $\mathrm{f}-$ monotone property,

(2.1.4) $\mathrm{f}$ is continuous and $\mathrm{f}$ commutes with $\mathrm{S}$,

(2.1.5) $f(X)$ is complete.

(2.1.6) (a) if a non decreasing sequence $\left\{x_{n}\right\} \rightarrow x$, then $x_{n} \leq x$ for all $n$,

(b) if a non increasing sequence $\left\{x_{n}\right\} \rightarrow x$, then $x \leq x_{n}$ for all $n$, for $\mathrm{x}_{0}, \mathrm{y}_{0}, \mathrm{z}_{0} \in \mathrm{X}$ there exists $\mathrm{x}_{1}, \mathrm{y}_{1}, \mathrm{z}_{1} \in \mathrm{X}$ such that

$\mathrm{fx}_{0} \geq \mathrm{S}\left(\mathrm{x}_{0}, \mathrm{y}_{0}, \mathrm{z}_{0}\right), \mathrm{fy}_{0} \leq \mathrm{S}\left(\mathrm{y}_{0}, \mathrm{x}_{0}, \mathrm{y}_{0}\right), \mathrm{fz}_{0} \geq \mathrm{S}\left(\mathrm{z}_{0}, \mathrm{y}_{0}, \mathrm{x}_{0}\right)$ and

$\mathrm{fx}_{1} \geq \mathrm{S}\left(\mathrm{x}_{1}, \mathrm{y}_{1}, \mathrm{z}_{1}\right), \mathrm{fy} \mathrm{y}_{1} \leq \mathrm{S}\left(\mathrm{y}_{1}, \mathrm{x}_{1}, \mathrm{y}_{1}\right), \mathrm{fz}_{1} \geq \mathrm{S}\left(\mathrm{z}_{1}, \mathrm{y}_{1}, \mathrm{x}_{1}\right)$ then $\mathrm{S}, \mathrm{T}, \mathrm{f}, \mathrm{g}$ have unique common tripled fixed point. 


\section{Conclusion}

In this article, the new Caristi type contraction in a Partially ordered metric space has been introduced and a fixed point theorem for four maps has been established with help of the new contraction. Our results is unified in the literature.

\section{Acknowledgement}

All authors are equally contributed in developing of this article. The authors are grateful to the learned referees for their careful reading of our manuscript.

\section{References}

[1] T.G.Bhaskar and V.Lakshmikantham, "Fixed point theorems in partially ordered metric spaces and applications", Nonlinear Analysis, 65, 2006, 1379-1393.

[2] V. Berinde and M. Borcut, " Tripled fixed point theorems for contraction type mappings in partially ordered metric spaces", Nonlinear Analysis, Volume 74(15), 2011, 4889-4897.

[3] K.P.R.Rao and G.N.V.Kishore, " A unique common tripled fixed point theorem in partially ordered cone metric spaces", Bulletin of mathematical Aanlyisi and Applications, Volume 3, 20112, 13-222.

[4] V. Lakshmikantham and Lj.Ciric "Coupled fixed point theorems for nonlinear contractions in partially ordered metric spaces", Nonlinear Analysis, 70, 2009, 4341-4349.

[5] J. S. Jung, Y. J. Cho, S. M. Kang, B. S. Lee, and Y. K. Choi "Coincidence point theorems in generating spaces of quasi metric family", Fuzzy Sets and Systems, Vol.116(3), 2000, 471-479.

[6] Caristi J, "Fixed point theorems for mappings satisfying in ward conditions", Transactions of the American Mathematical Society Vol. 215, 1976, pp. 241-251. 